\title{
Grado de severidad de la disfagia orofaríngea neurogénica en el paciente de unidad de cuidado intermedio
}

\begin{abstract}
Severity degree neurogenic oropharyngeal dysphagia in the patient of intermediate care unit
\end{abstract}
\author{
José José Ariza De La Barrera
}

\section{RESUMEN:}

Objetivo: Indagar el grado de severidad de la disfagia orofaríngea neurogénica en los pacientes de la unidad de cuidado intermedio, por medio de la escala de severidad de la disfagia de Horacio Cámpora que considera habilidades alimentarias a través de consistencias que puede manejar el paciente realizadas con el Método de Exploración Clínica Volumen - Viscosidad (MECV-V). Metodología: El estudio es de tipo exploratorio, no experimental, con un grupo de 32 pacientes con desórdenes neurológicos. Resultado: Se detecta que el grado de severidad de la disfagia orofaríngea neurogénica en los pacientes de la unidad de cuidados intermedios es moderada - severa, en la cual la ingesta oral no es exitosa y requiere supervisión constante y asistencia por el fonoaudiólogo. Conclusión: La investigación aporta elocuentemente al abordaje fonoestomatognatico y/o motricidad orofacial, en cuanto a la labor asistencial del fonoaudiólogo en las unidades de cuidados intermedios.

PALABRAS CLAVES: Disfagia orofaríngea neurogénica, desórdenes neurológicos, unidad de cuidados intermedios, fonoaudiólogo, fonoestomatognatico y/o motricidad orofacial.

\begin{abstract}
:
Purpose: The aim of this study is to investigate the degree of severity of neurogenic oropharyngeal dysphagia in patients of the intermediate care unit, using Horacio Campora's severity scale for dysphagia. This takes into account eating assestment for patients employing the volume-viscocity swallow test for clinical evaluation (V-VST). Methodology: The study, which is an exploratory research and not experimental, was performed on a group of 32 patients with neurological disorders. Results: A moderate -severe degree of neurogenic oropharyngeal dysphagia is detected in the patients of the intermediate care unit, where the oral intake of food/liquid is not successful. As a consequence, the patients require the constant assistance and supervision by a speech pathologist and language. Conclusion: The research contributes to the stomatognathic and/or orofacial motricity, regarding the assistance work of speech pathologist and language in the intermediate care unit.
\end{abstract}

KEYWORDS: Neurogenic oropharyngeal dysphagia, neurological disorders, intermediate care unit, speech pathologist and language, stomatognathic and/or orofacial motricity. 


\section{INTRODUCCIÓN}

La Unidad de Cuidados Intermedios San Luis S.A.S., es una unidad orgánica que brinda atención de salud para pacientes que previsiblemente tienen un bajo riesgo de necesitar medidas terapéuticas de soporte vital, pero que requieren más monitorización y cuidados de enfermería de los que pueden recibir en una planta de hospitalización convencional.

Además son pacientes estables, sin embargo, por la naturaleza de la enfermedad, son susceptibles de complicaciones que deben resolverse rápidamente. De allí nace dentro de esta entidad el departamento de rehabilitación, con miras a beneficiar la calidad de vida de los usuarios.

En dicha entidad existe un gran número de alteraciones neurológicas entre los cuales podemos encontrar traumatismos de cabeza o cuello, daño cerebral producto de tóxicos, enfermedades neurológicas como miastenia gravis, Parkinson, neoplasias y accidentes cerebrovasculares; que pueden presentar disfagia neurogénica.

La disfagia neurogénica es definida como la dificultad para tragar a causa de un desorden neurológico el cual altera las funciones sensoriales y motoras de las fases oral y faríngea de la deglución. La etapa esofágica difícilmente es afectada por alteraciones neurológicas, pero cuando lo es, suele ser un problema más sintomático al comparársele con los originados por disfunciones en las etapas oral y faríngea. (Buchholz, D., 1997). ${ }^{1}$

Así mismo, los daños en el sistema nervioso central pueden también resultar en disfagia oral y/o faríngea al envolver los sistemas aferentes; en los que se incluyen el complejo del núcleo trigémino, el núcleo del tracto solitario, las vías ascendentes en el tallo cerebral y subcorteza, estructuras subcorticales como el tálamo, y la corteza cerebral. ${ }^{2}$

Por otra parte, los daños en el sistema nervioso periférico asociado con disfagia oral y/o faríngea tienen como puntos potenciales de daño los nervios craneales inferiores, uniones neuromusculares y fibras musculares estriadas en la cavidad oral, lengua, faringe y laringe.

Por lo expuesto, el departamento de rehabilitación, específicamente del área de fonoaudiología por ser la disciplina que realiza el estudio del sistema fonoestomatognatico $\mathrm{y} / \mathrm{o}$ motricidad orofacial, evalúa funciones específicas de la deglución; por ello se plantea indagar el grado de severidad de la disfagia oro faríngea neurogénica en los pacientes de la unidad de cuidado intermedio, por medio de la escala de severidad de la disfagia de Horacio Cámpora que considera habilidades alimentarias a través de consistencias que puede manejar el paciente realizadas con el Método de Exploración Clínica Volumen Viscosidad (MECV-V).

\section{METODOLOGÍA}

El presente estudio es de tipo exploratorio, no experimental, debido a que se recolectaron datos en un solo momento y en un tiempo único con el propósito de conocer la severidad de una comunidad que presenta disfagia orofaríngea por problemas neurológicos.

El grupo en estudio se extrajo de los 60 usuarios que se encuentran en la Unidad de Cuidado Intermedio San Luis S.A.S., el cual corresponde a 32 pacientes que cumplieron con los tres criterios de inclusión descritos a continuación:

1. Obtención del correspondiente consentimiento informado de ellos o de sus representantes legales.

2. Pacientes con traumatismos de cabeza (trauma craneoencefálico) o cuello (trauma raquimedular en cervicales), daño cerebral por productos tóxicos, accidente $\mathrm{y} / \mathrm{o}$ evento cerebrovascular y enfermedades neurodegenerativas como Enfermedad de Alzheimer \& Parkinson.

3. Pacientes que poseen un mínimo estado de alerta que permita colaborar con el Método de Exploración Clínica Volumen - Viscosidad (MECV-V).

4. Pacientes que hablen o que realicen un sonido vocal para tener la voz inicial como control de cualquier alteración.

Teniendo en cuenta lo anterior se utilizó para este estudio la escala de grado de severidad de Horacio Cámpora obedeciendo a las manifestaciones alimentarias a través de consistencias que puede manejar el paciente realizadas con el Método de Exploración Clínica Volumen - Viscosidad (MECV-V), al cual se le agrego otra consistencia, la tipo miel para un mayor conocimiento de texturas en el grado de severidad de la disfagia. Está siendo una prueba sencilla y segura que indica los signos más frecuentes e importantes de la disfagia, obteniendo así información sobre la viscosidad y volumen más seguro para cada paciente. El MECV-V consiste en administrar al paciente 
diferentes volúmenes de alimento $5 \mathrm{ml}$ (bajo), $10 \mathrm{ml}$ (medio) y $20 \mathrm{ml}$ (alto) de alimento en texturas néctar, miel, pudding y líquido que se puede realizar con agua $\mathrm{y}$ un espesante.

Se inicia con la administración de $5 \mathrm{ml}$ de viscosidad néctar observando la posible presencia de los siguientes signos: Presencia de tos, cambios en el tono de voz, presencia de residuos orales, existencia de una deglución fraccionada y fallo del sello labial o residuos faríngeos.

Todo ello mientras se monitoriza la saturación de oxígeno (una disminución de la saturación basal de oxígeno $\geq 3 \%$ es un signo de aspiración) y realización de auscultación cervical.

\begin{tabular}{|c|c|c|c|c|c|c|c|c|c|c|c|c|}
\hline \multicolumn{13}{|c|}{ MÉTODO DE EXPLORACIÓN CLÍNICA VOLUMEN-VISCOCIDAD } \\
\hline \multicolumn{13}{|l|}{ NOMBRE: } \\
\hline EDAD: & \multicolumn{12}{|c|}{ FECHA DE APLICACIÓN: } \\
\hline \multicolumn{13}{|l|}{ SATURACION DE O2: } \\
\hline VISCOSIDAD & \multicolumn{3}{|c|}{ NÉCTAR } & \multicolumn{3}{|c|}{ MIEL } & \multicolumn{3}{|c|}{ LÍQUIDO } & \multicolumn{3}{|c|}{ PUDDING } \\
\hline VOLUMEN & $\begin{array}{l}5 \\
\mathrm{ML}\end{array}$ & $\begin{array}{l}10 \\
\mathrm{ML}\end{array}$ & $\begin{array}{l}20 \\
\mathrm{ML} \\
\end{array}$ & \begin{tabular}{|l}
5 \\
$\mathrm{ML}$ \\
\end{tabular} & $\begin{array}{l}10 \\
\mathrm{ML}\end{array}$ & \begin{tabular}{|l|}
20 \\
$M L$
\end{tabular} & \begin{tabular}{|l}
5 \\
$\mathrm{ML}$ \\
\end{tabular} & $\begin{array}{l}10 \\
\mathrm{ML}\end{array}$ & \begin{tabular}{|l|}
20 \\
$\mathrm{ML}$
\end{tabular} & \begin{tabular}{|l}
5 \\
$\mathrm{ML}$ \\
\end{tabular} & $\begin{array}{l}10 \\
\text { ML }\end{array}$ & $\begin{array}{l}20 \\
\text { ML }\end{array}$ \\
\hline \multicolumn{13}{|c|}{ ALTERACIONES O SIGNOS DE SEGURIDAD } \\
\hline \multicolumn{13}{|l|}{ TOS } \\
\hline \multicolumn{13}{|l|}{ CAMBIO DE VOZ } \\
\hline \multicolumn{13}{|l|}{ DESATURACIÓN DE O2 } \\
\hline \multicolumn{13}{|c|}{ ALTERACIONES O SIGNOS DE EFICACIA } \\
\hline \multicolumn{13}{|l|}{ SELLO LABIAL } \\
\hline \multicolumn{13}{|l|}{ RESIDUO ORAL } \\
\hline \multicolumn{13}{|l|}{ DEGLUCIÓN FRACIONADA } \\
\hline \multicolumn{13}{|l|}{ RESIDUO FARÍNGEA } \\
\hline \multicolumn{13}{|c|}{ GRADO DE SEVERIDAD DE LA DISFAGIA } \\
\hline \multicolumn{13}{|l|}{ NORMAL } \\
\hline \multicolumn{13}{|l|}{ LEVE } \\
\hline \multicolumn{13}{|l|}{ MODERADA } \\
\hline \multicolumn{13}{|l|}{ MODE - SEVERA } \\
\hline \multirow{2}{*}{\multicolumn{13}{|c|}{ INGESTA DE FLUIDOS RECOMENDADOS }} \\
\hline & & & & & & & & & & & & \\
\hline VISCOSIDAD & \multicolumn{3}{|c|}{ NECTAR } & \multicolumn{3}{|c|}{ MIEL } & \multicolumn{3}{|c|}{ LIQUIDO } & \multicolumn{3}{|c|}{ PUDDING } \\
\hline VOLUMEN & \multicolumn{4}{|c|}{ BAJO } & \multicolumn{4}{|c|}{ MEDIO } & & & LTO & \\
\hline $\begin{array}{l}\text { EVALUACION FINAL: } \\
\text { Disfagia: }\end{array}$ & & & & & & & & & & & & \\
\hline RECOMENDACIONES: La & ción d & $\begin{array}{r}\text { fluidos } \\
\mathrm{y} \text { vo }\end{array}$ & $\begin{array}{l}\text { debe re } \\
\text { lumen }\end{array}$ & alizarse & a visc & sidad & & & & & & \\
\hline$\%$ de la Disfagia $=\underline{\text { Valor Fi }}$ & $\begin{array}{ll}\text { Inicic } \\
\text { Final }\end{array}$ & $\times 100=$ & & & & & & & & & & \\
\hline
\end{tabular}

Figura 1. Hoja de Registro del MECV-V. 
Esta prueba detecta muy bien la eficacia de la deglución valorando como prueba positiva cualquiera de estos tres aspectos: 1). El sello labial: incapacidad de mantener el bolo dentro de la boca. 2). La existencia de residuos orales en la lengua o debajo de la misma, en encías o cuando se sospecha de residuos en la faringe. 3). Deglución fraccionada.

También detecta bien la seguridad ya que si durante la misma se presenta alguna de las alteraciones antes descritas (tos etc.), se debe determinar como positiva la prueba y aumentar la viscosidad al nivel siguiente y /o disminuir el volumen.

Para la realización del método clínico de volumenviscosidad (MECV-V) se necesitan los siguientes materiales: Agua a temperatura ambiente, espesante (Enterex Espesante Polvo - Lab. Victus), jeringa de 50ml, 4 vasos (para las distintas viscosidades), pulsioxímetro, fonendoscopio, hoja de registro (Fig. $1)$.
Procedimiento. La valoración se realiza con el paciente en sedestación y monitorización continua de la saturación de oxígeno (Pulsioxímetro). Se administran cuencos de 5, 10 y $20 \mathrm{ml}$ con viscosidades néctar, miel, pudding y líquida (agua). Se inicia por la viscosidad néctar a volumen bajo, para ir aumentando el volumen, y se sigue el mismo procedimiento con el miel, luego líquido claro (agua) y, finalmente, con la viscosidad pudding. En cada textura / volumen se registra si se producen signos de alteración de la seguridad (tos, descenso de la saturación basal de oxígeno mayor de un 5\% y cambio del tono en la voz) $\mathrm{y} / \mathrm{o}$ de la eficacia (sellado labial insuficiente, residuos orales o faríngea y deglución fraccionada).Cuando observamos signos que comprometen la seguridad del paciente, no se pasa ni a un volumen más grande ni a una viscosidad inferior. Se concluye que no existe disfagia cuando no se evidencian signos de alteración en la seguridad ni en la eficacia en ningún momento de la prueba. ${ }^{3}$

\begin{tabular}{|c|l|}
\hline GRADO & \multicolumn{1}{|c|}{ DETALLE } \\
\hline Normal & $\begin{array}{l}\text { Masticación y deglución segura, eficiente en todas las consistencias de los } \\
\text { alimentos. }\end{array}$ \\
\hline Leve & $\begin{array}{l}\text { Masticación y deglución eficiente en la mayoría de los alimentos. } \\
\text { Ocasionalmente puede presentar dificultad. Requiere del uso de técnicas } \\
\text { específicas para lograr una deglución satisfactoria. }\end{array}$ \\
\hline Moderada & $\begin{array}{l}\text { Deglución aceptable con dieta blanda, pero puede tener dificultad con } \\
\text { líquidos y sólidos. Requiere supervisión y tratamiento. }\end{array}$ \\
\hline Moderadamente Severa & $\begin{array}{l}\text { Paciente cuya ingesta oral no es exitosa. Requiere supervisión constante y } \\
\text { asistencia. Sólo puede alimentarse con terapista. }\end{array}$ \\
\hline Severa & $\begin{array}{l}\text { La nutrición del paciente es por método alternativo. No ingiere alimento } \\
\text { por boca. Estos trastornos deglutorios se deben a alteraciones tanto de } \\
\text { la motilidad como de la sensibilidad, o a la combinación de ambos, } \\
\text { produciéndose una falta de coordinación y cumplimiento de las distintas } \\
\text { etapas del proceso deglutorio. }\end{array}$ \\
\hline
\end{tabular}

Figura 2. Escala de Severidad de la disfagia de Horacio Cámpora.

\begin{tabular}{|l|c|c|c|c|c|c|c|}
\hline \multirow{2}{*}{ CRITERIOS } & \multicolumn{2}{c|}{ TC } & \multirow{2}{*}{ DCT } & \multirow{2}{*}{ ACV } & \multicolumn{2}{|c|}{ END } & \multirow{2}{*}{ TOTAL } \\
\cline { 2 - 4 } & TCE & TRC & & & EA & EP & \\
\hline ALTERACIONES O SIGNOS DE EFICACIA & & & & \\
\hline SELLO LABIAL & 1 & 0 & 0 & 4 & 0 & 0 & 5 \\
\hline RESIDUO ORAL & 3 & 1 & 0 & 4 & 1 & 1 & 10 \\
\hline $\begin{array}{l}\text { DEGLUCIÓN } \\
\text { FRACIONADA }\end{array}$ & 2 & 1 & 1 & 5 & 2 & 0 & 11 \\
\hline RESIDUO FARÍNGEA & 1 & 0 & 0 & 3 & 1 & 1 & 6 \\
\hline TOTAL POBLACIÓN & 7 & 2 & 1 & 16 & 4 & 2 & 32 \\
\hline
\end{tabular}

Figura 3. Alteraciones o Signos de Eficacia. 
Por otro lado la Escala de Severidad de la disfagia de Horacio Cámpora ${ }^{4}$ (Fig. 2), considera las habilidades alimentarias a través de las consistencias que puede manejar el paciente con prueba de alimentos sin utilización de videofluoroscopía.

Esta se aplicara durante la ingesta de las cuatro consistencias (néctar, miel, pudding y liquida) que se manejaran con el Método de Exploración Clínica Volumen - Viscosidad (MECV-V), con el fin de indagar el grado de severidad de la disfagia orofaríngea neurogénica en la unidad de cuidado intermedio.

\section{RESULTADOS}

Contribuyendo al desarrollo del estudio de la deglución, a continuación se presenta la distribución del tamaño de la muestra $(\mathrm{n}=32)$, está distribuido de la siguiente forma:

$\begin{array}{llr} & \begin{array}{l}\text { Trauma } \\ \text { _ Traumatismos de }\end{array} & \text { Craneoencefálico } \\ \text { Cabeza } & \text { Trauma Raquimedular } & \\ & \text { Cervical } & 2 \\ \text { - Daño Cerebral por Productos Tóxicos } & 1 \\ \text { - Accidente y/o Evento Cerebrovascular } & 16 \\ & \text { Enfermedad de } \\ \text { - Enfermedades } & \text { Alzheimer } \\ \text { Neurodegenerativas } & \text { Enfermedad de } \\ & \text { Parkinson } & \\ & \end{array}$

En la indagación a través de consistencias que puede manejar los pacientes realizados con el Método de Exploración Clínica Volumen - Viscosidad (MECV-V) se obtuvo:

1. En las alteraciones o signos de seguridad se obtuvo que 17 pacientes de 32 , presentan tos al momento de deglutir el alimento, acompañado en la mayoría de los casos por cambios en las cualidades de la voz. (Fig. 3)

2. En las alteraciones o signos de eficacia en la deglución se evidencia que 11 pacientes de 32, presentan una deglución fragmentada en la fase orofaríngea, seguido por residuos en cavidad oral que puede ser el desencadenador de la deglución fragmentada. (Fig. 4)

3. En el grado de severidad de la disfagia se evidencia que 17 usuarios de 32, presentan una alteración en la deglución de moderada a severa. (Fig. 5)

4. En cuanto a la ingesta de fluidos recomendados, a nivel de viscosidad el mayor riesgo se presenta a nivel de líquidos con 14 pacientes de 32, seguido de néctar (8), miel (6) y pudding (4). En cuanto a volumen el mayor riesgo está en la ingesta de $5 \mathrm{ml}$ en cualquier tipo de consistencia. (Fig. 5)

De lo anterior, se resalta que la mayor parte de la población en estudio que se le aplico el MECV-V presentaba un accidente o evento cerebro vascular.

Ahora según los resultados obtenidos con el MECV-V, ubicándolos en la escala de severidad de la disfagia de Horacio Cámpora, se detecta que el grado de severidad de la disfagia orofaríngea neurogénica en los pacientes de la unidad de cuidados intermedios es moderada - severa, en la cual ingesta oral no es exitosa y requiere supervisión constante y asistencia por el fonoaudiólogo.

Teniendo como mayor diagnostico una: Disfagia orofaríngea neurogénica de severidad moderada severa para consistencias liquidas y moderada para tipo néctar; caracterizada por constantes tos, cambios en la voz, residuos en cavidad oral y deglución fragmentada, secundario a un accidente o evento cerebro vascular.

\begin{tabular}{|l|c|c|c|c|c|c|c|}
\hline \multirow{2}{*}{ CRITERIOS } & \multicolumn{2}{c|}{ TC } & \multirow{2}{*}{ DCT } & \multirow{2}{*}{ ACV } & \multicolumn{2}{c|}{ END } & \multirow{2}{*}{ TOTAL } \\
\cline { 2 - 3 } & TCE & TRC & & & EA & EP & \\
\hline GRADO DE SEVERIDAD DE LA DISFAGIA \\
\hline NORMAL & 0 & 0 & 0 & 0 & 0 & 0 & 0 \\
\hline LEVE & 0 & 0 & 0 & 0 & 0 & 0 & 0 \\
\hline MODERADA & 4 & 0 & 1 & 5 & 1 & 0 & 11 \\
\hline MODE - SEVERA & 3 & 2 & 0 & 7 & 3 & 2 & 17 \\
\hline SEVERA & 0 & 0 & 0 & 4 & 0 & 0 & 4 \\
\hline TOTAL POBLACIÓN & 7 & 2 & 1 & 16 & 4 & 2 & 32 \\
\hline
\end{tabular}

Figura 4. Grado de Severidad de la Disfagia. 


\begin{tabular}{|c|c|c|c|c|c|c|c|c|}
\hline \multirow{2}{*}{\multicolumn{2}{|c|}{ CRITERIOS }} & \multicolumn{2}{|c|}{$\mathrm{TC}$} & \multirow{2}{*}{ DCT } & \multirow[t]{2}{*}{$\mathrm{ACV}$} & \multicolumn{2}{|c|}{ END } & \multirow{2}{*}{ TOTAL } \\
\hline & & TCE & TRC & & & EA & EP & \\
\hline \multicolumn{9}{|c|}{ INGESTA DE FLUIDOS RECOMENDADOS } \\
\hline \multirow{4}{*}{ VISCOSIDAD } & NECTAR & 1 & 2 & 0 & 4 & 1 & 0 & 8 \\
\hline & MIEL & 2 & 0 & 0 & 3 & 1 & 0 & 6 \\
\hline & LIQUIDO & 4 & 0 & 1 & 5 & 2 & 2 & 14 \\
\hline & PUDDING & 0 & 0 & 0 & 4 & 0 & 0 & 4 \\
\hline \multicolumn{2}{|c|}{ TOTAL POBLACIÓN } & 7 & 2 & 1 & 16 & 4 & 2 & 32 \\
\hline \multirow{3}{*}{ VOLUMEN } & BAJO & 4 & 0 & 1 & 9 & 2 & 2 & 18 \\
\hline & MEDIO & 3 & 2 & 0 & 6 & 1 & 0 & 12 \\
\hline & ALTO & 0 & 0 & 0 & 1 & 1 & 0 & 2 \\
\hline \multicolumn{2}{|c|}{ TOTAL POBLACIÓN } & 7 & 2 & 1 & 16 & 4 & 2 & 32 \\
\hline
\end{tabular}

Figura 5. Ingesta de fluidos recomendados.

\section{DISCUSIÓN}

De acuerdo a los resultados obtenidos y en comparación con la literatura en torno a la disfagia neurogénica en pacientes dentro de las unidades de cuidados intermedios, la mayor incidencia son los accidentes o eventos cerebrovasculares en comparación con el resto de alteraciones neurológicas.

Al parecer hay numerosos estudios que evidencian una relación entre accidentes cerebro vascular con trastornos de la deglución. (Veis, S. y Logemann, J., 1985; ${ }^{5}$ Wade, D. y Hewer, R., 1987; ${ }^{6}$ Horner, J. y Cols., 1991; ${ }^{7}$ Albert, M. y Cols., 1992; ${ }^{8}$ Daniels, S. y Foundas, A., 1997; ${ }^{9}$ Hamdy, S. y Cols., 2000; 10 Paciaroni, M. y Cols., $2004{ }^{11}$ ).

Como se evidencia en la investigación al indagar el grado de severidad de la disfagia orofaríngea neurogénica en los pacientes de la unidad de cuidado intermedio, por medio de la escala de severidad de la disfagia de Horacio Cámpora utilizando el Método de Exploración Clínica Volumen - Viscosidad (MECV-V); la mayor prevalencia es el ACV. Teniendo como sesgo el resto de patologías puesto que su prevalencia es inferior en la investigación.

\section{CONCLUSIONES}

Los resultados de la investigación aportan elocuentemente al abordaje fonoestomatognatico y/o motricidad orofacial, en cuanto a la labor asistencial del fonoaudiólogo en las unidades de cuidados intermedios, debido que el grado de severidad de la disfagia orofaríngea neurogénica en los pacientes que se encuentran en ellas es moderada - severa, en la cual la ingesta oral no es exitosa y requiere supervisión constante en el proceso motor de la función oralfaríngea.

Además, para una adecuada intervención y rehabilitación de la disfagia orofaríngea neurogénica de severidad moderada - severa en las unidades de cuidad intermedio, es indispensable el fonoaudiólogo, quien realiza el manejo de habilidades alimentarias a través de consistencias que puede manejar el paciente, teniendo en cuenta:

1. La definición de objetivos a corto y a largo plazo, los cuales deberán ser revisados de acuerdo con la revaloración periódica,

2. La orientación al personal de enfermería acerca del desorden deglutorio,

3. La revisión y seguimiento periódico de las metas y logros alcanzados,

4. La revaloración del estado actual del paciente (ajustes pertinentes),

5. El tratamiento puede realizarse en simultánea con la intervención de alteraciones del lenguaje y/o de la función comunicativa,

6. La realización de remisiones pertinentes y oportunas adjuntando informes de la valoración, 
7. Y deberá tenerse en cuenta la interdisciplinariedad y transdisciplinariedad para la planeación y tratamiento de las discapacidades.

\section{Correspondencia}

José José Ariza De La Barrera

Fonoaudiólogo - Universidad de Sucre

Unidad de Cuidados Intermedio San Luis S.A.S.

Calle 144 \# 46- 41 Bogotá D.C. (Colombia)

(+57) 3045445300 -jjarba@gmail.com

\section{REFERENCIAS BIBLIOGRÁFICAS}

1. Buchholz D. Neurilogic disorders of swallowing. Dysphagia: diagnosis and management. Massachusetts: Butterworth - Heinemann [Internet] 1997, Oct. Winter; 10 (1): pp. 37 - 72. Disponible desde: http://www.ncbi.nlm.nih.gov/pubmed/ 7859526

2. Ertekin C,Aydogdu I. Neurophisiology of swallowing. Clinical Neurophisiology, 2003, Ene. 114 (1), pp. $2226-2244$.

3. Ferrero López MI, Castellano Vela E, Navarro Sanz R. Utilidad de implantar un programa de atención a la disfagia en un Hospital de Media y Larga Estancia. Scielo Nutrición Hospitalaria 24 (5) 2009, Sep.- Oct. Madrid - España. Disponible desde: http://scielo.isciii.es/scielo.php?pid=S0212$16112009000500011 \&$ script $=$ sci_arttext

4. Araya C. Evaluación de deglución en pacientes con accidente vascular encefálico agudo. Universidad de Chile. [Internet]. Procedimiento, 2004, [Tesis]. Pp. 37 - 42. Disponible desde: http://repositorio.uchile. cl/tesis/uchile/2004/florin_c/doc/florin_c.pdf
5. Veis S, Logemann J. Swallowing disorders in persons with cerebrovascular accident. Archives of Physical Medicine and Rehabilitation, 1985, Feb., 66: pp. 372 -375 .

6. Wade D, Hewer R. Motor loss and swallowing difficulty after stroke: frequency, recovery and prognosis. Acta neurológica Scandinavica, 1987, Dic. 76 (1): pp. $50-54$.

7. Horner J, Buoyer F, Alberts J, Helms M. Dysphagia following brain - stem stroke: clinical correlates and outcome. Archives of Neurology 1991, Dic. 48: pp. $1170-1173$.

8. Albert M, Horner J, Gray L, Brazer S. Aspiration after stroke: lesion analysis by brain MRI. Dysphagia [Internet] 1992, Sep., 7: pp. 170 - 173. Disponible desde: http://link.springer.com/article/10.1007/ BF02493452

9. Daniels S, Foundas A. The role of the insular cortex in dysphagia. Dysphagia [Internet] 1997, May., 12: pp. 146 - 156. Disponible desde: http://link.springer. com/article/10.1007/PL00009529

10. Hamdy S, Rothwell J, Aziz Q, Thompson D. The organisation and reorganisation of human swallowing motor cortex: implications for recovery after stroke. Clinical Science [Internet] 2000, Jul., 98: pp. 151 - 157. Disponible desde: http://www.clinsci.org/ content/97/s41/10P.3

11. Paciaroni M, Mazzotta G, Corea F, Caso V, Venti M, Milia P, Silvetrelli G, Plamerini F, Parnetti L, Gallai V. Dysphagia following stroke.. European Neurology [Internet] 2004, May. 51: pp. 162 - 167. Disponible desde: http://www.karger.com/Article/Abstract/ 77663 